OPEN ACCESS

Edited by:

Emiliano Cè,

University of Milan, Italy

Reviewed by:

Vitor Pereira Costa,

Santa Catarina State University, Brazil

Gleber Pereira

Federal University of Paraná, Brazi

Daniel Gomes Da Silva Machado,

Federal University of Rio Grande do

Norte, Brazil

${ }^{*}$ Correspondence:

Erik Borg Kolsung

kolsung@hotmail.com

Specialty section:

This article was submitted to

Exercise Physiology,

a section of the journal

Frontiers in Physiology

Received: 05 May 2020

Accepted: 10 August 2020

Published: 27 August 2020

Citation:

Kolsung EB, Ettema $G$ and Skovereng K (2020) Physiological Response to Cycling With Variable

Versus Constant Power Output.

Front. Physiol. 11:1098.

doi: 10.3389/fphys.2020.01098

\section{Physiological Response to Cycling With Variable Versus Constant Power Output}

\author{
Erik Borg Kolsung*, Gertjan Ettema and Knut Skovereng
}

Centre for Elite Sports Research, Department of Neuromedicine and Movement Science, Norwegian University of Science and Technology, Trondheim, Norway

Introduction: Variable power output (VP) is one of the main characteristics of a road cycling mass-start. Tolerating VP during outdoor road cycling highly influences performance. There is a lack of continuous and comprehensive measurements during this power condition. Accordingly, the aim of the present study was to investigate physiological response to VP vs. constant power output (CP) as well as the perceived exertion of these two power conditions, and to investigate if variations in power output which span above lactate threshold (LT), differ from variations below LT.

Methods: 15 elite competitive cyclists completed three test days, including 1 day of baseline testing and 2 days of main testing, consisting of four bouts of 28 min at two different intensities, "low" at 70\% of LT and "high" at 95\% of LT, with VP and CP. VP was performed with a 15\% fluctuation of the average power output every second minute. Maximal oxygen uptake $\left(\mathrm{VO}_{2}\right)$, respiratory exchange ratio (RER), heart rate $(\mathrm{HR})$, blood lactate (LA), rating of perceived exertion (RPE), cadence (RPM) and power output (W) were measured.

Results: At both low and high intensity, the VP condition induced a significantly higher $\mathrm{VO}_{2}, \mathrm{HR}$ and LA than the CP condition. Whole-bout RPE was similar between power conditions at high intensity. Additionally, at the high intensity, cycling with VP led to a greater increase in LA and lesser increase in RPE compared to cycling with CP.

Discussion: The results of this study show that, despite considerable differences in the demand during the VP and CP bouts, there are minor differences in the perceptual and physiological response directly following these two power conditions in a cohort of elite competitive cyclists. A practical implication of these findings is that training with VP seems to be a viable alternative to training with $\mathrm{CP}$, at least at high intensity.

Keywords: cycling, performance, variable power, constant power, physiological response

\section{INTRODUCTION}

One of the main characteristics of competitive cycling is variations in power output (Faria et al., 2005; Ebert et al., 2006) which occur as a consequence of changing weather conditions, changing terrain and the group dynamics of the peloton (e.g., drafting) (Palmer et al., 1994). To perform well and to win races, cyclists need to be able to tolerate variations in power output. The effects 
of a variable power output (VP) versus constant power output (CP) have previously been studied, both in terms of pacing strategies (Atkinson and Brunskill, 2000), subsequent performance (Palmer et al., 1997), and isolated physiological response (Liedl et al., 1999). In a time-trial, a pacing strategy with constant velocity produces the best performances (Foster et al., 1993; Swain, 1997; Atkinson and Brunskill, 2000), meaning that a CP strategy is superior when the course is completely flat and without wind (e.g., track cycling). In contrast, mathematical models have demonstrated that increasing power output with as little as $5 \%$ when riding uphill or into headwind, and a corresponding decrease when riding downhill or in tailwind, will lead to a better time-trial performance than keeping the power output constant (Swain, 1997; Wells and Marwood, 2016).

Training with CP is common among cyclists and other endurance athletes. Traditional interval training during the preparation period often consist of longer work bouts $(\sim 8-$ $15 \mathrm{~min}$ ) with $\mathrm{CP}$, with a work rate at or slightly below the lactate threshold (LT) (Solli et al., 2017; Rønnestad and Hansen, 2018). Considering the training principle of specificity, training with VP may, in many cases, mimic race situations to a greater extent than training with $\mathrm{CP}$. Even though designing training programs concerns more than just the principle of specificity, it could be argued that cyclists advantageously could include more VP work into their training. However, if the physiological cost of VP differs from CP, careful considerations regarding total training load and periodization of these workouts would be necessary when designing training programs.

Liedl et al. (1999) reported that no additional physiological cost in terms of $\mathrm{VO}_{2}$, heart rate and lactate was found after a 1-h effort performed with VP compared to the same effort performed with CP. Similarly, Brickley et al. (2007) reported that variations in power output did not have a significant effect on muscle metabolism. However, elevated blood lactate (LA) levels during VP compared to CP has previously been reported (Palmer et al., 1999; Suriano et al., 2007) and greater power variation have been shown to increase neuromuscular fatigue (Theurel and Lepers, 2008).

Although the knowledge is growing on the physiological response to VP and $\mathrm{CP}$, there is a lack of continuous and comprehensive measurements of performance related variables [e.g., LA, oxygen consumption $\left(\mathrm{VO}_{2}\right)$, metabolic rate $(\mathrm{MR})$ etc.] during VP and CP. Previous studies have neither investigated physiological response in competitive elite cyclists, which may be more accustomed to variations in power output from their racing experience, nor looked at VP and CP differences when performing low intensity exercise, which cyclists do to a large extent.

The training intensity distribution of elite endurance athletes, including cyclists, is approximately $80 \%$ low-intensity (LIT) and 20\% moderate- (MIT) and high-intensity (HIT) (Seiler and Tønnesen, 2009; Seiler, 2010). Additionally, as much as $40-45 \%$ of flat and hilly mass-start stages of the Tour Down Under (Ebert et al., 2006) was spent at an intensity that could be classified as LIT (i.e., $<1.9 \mathrm{~W} / \mathrm{kg}$ ). While the power output during the large LIT volume in training can be controlled and quite constant, the power output corresponding to LIT during races would include more variation due to the previously mentioned peloton dynamics. However, if CP and VP during LIT differs in physiological and perceptual cost has, to the best of our knowledge, not been investigated.

Accordingly, the primary aim of the present study was to investigate physiological and perceptual response to VP vs. CP in elite competitive cyclists. The secondary aim was to investigate if variations in power output which span above LT, differ from variations below LT. Based on the exponential nature of LA accumulation above LT (Faude et al., 2009), the substantial change in $\mathrm{VO}_{2}$ kinetics above LT, including the slow component (Lucia et al., 2000), and the findings of previous studies with their respective protocols, the hypothesis of the present study was that cycling with a VP condition would induce higher mean physiological cost in terms of $\mathrm{VO}_{2}, \mathrm{HR}$, and $\mathrm{LA}$ as well as a higher RPE, than a CP condition, but only at the high intensity where the power variation fluctuated above and below LT.

\section{MATERIALS AND METHODS}

\section{Participants}

19 elite competitive male cyclists (Table 1) were recruited through local clubs and teams in the area of Trondheim, Norway. Four of the participants were excluded - three of them due to measurement errors and one of them because he was not able to complete the full protocol. The study was conducted in the preparation period (i.e., November to January) for elite competitive cyclists. All of the participants were experienced with bike racing and seven of the participants had competed in races categorized by the Union Cycliste Internationale (UCI) as category 1.2 or higher. The participants were informed verbally about the procedures and purpose of the study and signed an informed written consent prior to participation. When explaining the study, we only stated that we wanted to compare VP and CP at high and low intensity, and we made sure not to mention our hypothesis about the study. The study was performed according to the Helsinki Declaration of 1975 and was registered and approved by Norwegian Social Science Data Services.

\section{Experimental Protocol}

Each participant came to the laboratory on three occasions at a standardized time of day, each preceded by at least 1 day of low intensity, short-duration exercise. Each participant completed the three test days within $6 \pm 4$ days.

The first day of testing was baseline testing consisting of a lactate profile test and $\mathrm{VO}_{2 \max }$ test (Sylta et al., 2016). After the measurement of height and body mass, the participants were allowed a 10 -min warm-up with freely chosen cadence at an intensity $<70 \%$ of LT before the lactate profile test started at a power output of $125 \mathrm{~W}$ with $50 \mathrm{~W}$ increases every $5 \mathrm{~min}$. After reaching a LA of $2 \mathrm{mmol} / \mathrm{l}$, work rate increments were reduced to $25 \mathrm{~W}$ and the test was terminated when LA exceeded $4 \mathrm{mmol} / \mathrm{l}$. A lactate sample was taken at the end of every step and the power output corresponding to $4 \mathrm{mmol} / \mathrm{l}$ was determined as LT. The protocol that we used to determine power output at $4 \mathrm{mmol} / \mathrm{l}$ is a known protocol for our participants, with many of them having 
TABLE 1 | Characteristics of the 15 included cyclists (mean \pm SD)

\begin{tabular}{lc}
\hline Age (years) & $\mathbf{2 4 . 9} \pm \mathbf{7 . 6}$ \\
\hline Weight $(\mathrm{kg})$ & $72.6 \pm 7.3$ \\
Height $(\mathrm{cm})$ & $182.2 \pm 7.1$ \\
$\mathrm{VO}_{2 \max }(\mathrm{ml} / \mathrm{kg} / \mathrm{min})$ & $72.9 \pm 5.1$ \\
$\mathrm{VO}_{2 \max }(\mathrm{L} / \mathrm{min})$ & $5.3 \pm 0.4$ \\
$\mathrm{LT}(\mathrm{W})$ & $310.5 \pm 21.7$ \\
$\mathrm{LT}(\mathrm{W} / \mathrm{kg})$ & $4.3 \pm 0.4$ \\
$\mathrm{PPO}(\mathrm{W})$ & $415.0 \pm 28.0$ \\
$\mathrm{PPO}(\mathrm{W} / \mathrm{kg})$ & $5.8 \pm 0.5$ \\
Number of races last season & $33.9 \pm 17.2$ \\
Training volume last season (hours) & $691.0 \pm 186.6$ \\
\hline LT lactate threshold: $P P O$, peak power output: $\mathrm{VO}$. & maximal oxygen uptake
\end{tabular}

performed it several times each year during the past years. Having the $4 \mathrm{mmol} / \mathrm{l}$ protocol in our study, we also gave something back to the participants, i.e., they used the results of the first test day in their own training prescription/evaluation. The protocol has also previously been used in other studies (Sylta et al., 2016).

Following a 10 -min active recovery with a freely chosen cadence and an intensity $<70 \%$ of $\mathrm{LT}$, a $\mathrm{VO}_{2 \max }$ test was performed. No specific measurements of recovery from the lactate profile test was taken. The $\mathrm{VO}_{2 \max }$ test started at the same load as the penultimate stage from the lactate profile test, and work rate was increased by $25 \mathrm{~W}$ every minute until volitional exhaustion while given strong verbal encouragement. Peak power output was calculated as the mean work rate during the final minute of the test and $\mathrm{VO}_{2 \max }$ was calculated as the highest 1-min average during the test. $\mathrm{VO}_{2}$, heart rate and cadence were measured continuously, and blood lactate was measured 1 min after the completion of the test. Considering that the participants in this study were elite and that they were familiar with the lactate profile test and the $\mathrm{VO}_{2 \max }$ test in a combined protocol, we are confident that the recovery time between the two tests was sufficient.

The second and third day of testing started with a 25-min warm-up at low intensity ( $<70 \%$ of LT) with freely chosen cadence, followed by a low intensity bout and a high intensity bout with VP or $\mathrm{CP}$, separated by $10 \mathrm{~min}$ of active recovery (freely chosen cadence and $<70 \%$ of LT) (Figure 1). All bouts included an identical 5 min incremental ramp, a 20 min main period with work matched CP or VP, and a final $3 \mathrm{~min}$ at $95 \%$ of LT. The $3 \mathrm{~min}$ at $95 \%$ of LT prior to and after each of the four conditions were done in order to compare the effects of the different bouts at a standardized load and was not meant to directly reflect the high and low conditions, respectively. The high and low conditions were performed at an average power output of $95 \%$ and $70 \%$ of LT, respectively, and the VP condition was performed with a $15 \%$ fluctuation every second minute. This $15 \%$ amplitude of fluctuation allowed the highest intensity to reach $110 \%$ of LT and thus achieve our aim of a condition above steady state. The $2 \mathrm{~min}$ duration of fluctuations was chosen in order to allow for changes in physiological variables to occur, while not being so long that the highest intensity would be exhaustive.
All bouts were performed with a freely chosen cadence and in a seated position. The cyclists were allowed only short periods of standing $(<10 \mathrm{~s})$ for comfort reasons. The time points for the LA and RPE measurements are shown in Figure 1. RPE was measured using Borg's 6-20 RPE scale and the participants were instructed to rate their whole-body feeling of exertion at the current time of measurement. All participants were familiar with the Borg's scale both from prior use and from the first day prior to the second and third day of testing. Whole-bout RPE-score was measured straight after the final $3 \mathrm{~min}$ at $95 \%$ of LT in each bout and the participants were instructed to rate their level of exertion for the bout as a whole.

The low intensity bout was performed before the high intensity bout, due to the expectation of a lesser influence from the low intensity bout on the high intensity bout than the opposite order. The order of power strategy (VP or CP) was randomized, which mean that the participants were randomized into 4 possible orders, i.e., low variable (LV) or low constant (LC) followed by high variable (HV) or high constant (HC) on day 1 , and then the remaining two bouts on day 2 . The distribution across the 4 possible randomization orders for our 15 included participants was 3, 4, 5, and 3 athletes, respectively. Food and drinks were allowed in between the two bouts. The participants were instructed to replicate quantity and type of food and drink intake from day 2 to day 3 of testing.

\section{Equipment and Measurements}

All testing was performed in a laboratory with stable conditions (temperature $\sim 18^{\circ} \mathrm{C}$ and relative humidity $\sim 30 \%$ ). The cyclists used their own road bike on an electronically braked indoor cycle trainer (CompuTrainerTM, RacerMate ${ }^{\circledR}$ Inc., Seattle, United States) calibrated according to the manufacturer's instruction and using a standardized tire pressure of 7 bar. An electrical fan was made available during all tests. A $20 \mu \mathrm{l}$ blood sample was collected from the participant's fingertip and LA was analyzed using the Biosen C-Line Sport lactate measurement system (EKF Industrial Electronics, Magdeburg, Germany). HR was measured with a heart rate monitor (Garmin Forerunner 920XT, Garmin International Inc., Kansas, United States). $\mathrm{VO}_{2}$ and RER were measured with an open-circuit indirect calorimetry apparatus (Oxycon Pro, Jaeger GmbH, Hoechberg, Germany). The apparatus was calibrated on every test day, using a gas of known concentration $\left(15.0 \% \mathrm{O}_{2}\right.$ and $5.0 \% \mathrm{CO}_{2}$, RiessnerGase $\mathrm{GmbH} \&$ Co., Lichtenfels, Germany) and a 3-liter syringe (Hans Rudolph Inc., Kansas City, MO, United States). $\mathrm{VO}_{2}$, RER, $\mathrm{HR}, \mathrm{RPM}$, and W were continuously measured throughout all the four bouts during main testing.

\section{Data Analysis}

Mean $\mathrm{VO}_{2}$, RER, HR, and LA were calculated for the whole 20min main period and for the last minute average for every high and low power output segment during the $20 \mathrm{~min}$ period of VP and the corresponding segments of CP (e.g., the first upper power segment of the VP bout was from 05:00 to 07:00, so the time 06:00-07:00 was used in the $\mathrm{VO}_{2}, \mathrm{RER}$ and $\mathrm{HR}$ calculation for both the VP and CP). We expected more variations in LA during VP compared to CP (Beneke, 2003), thus, LA was sampled more 
1

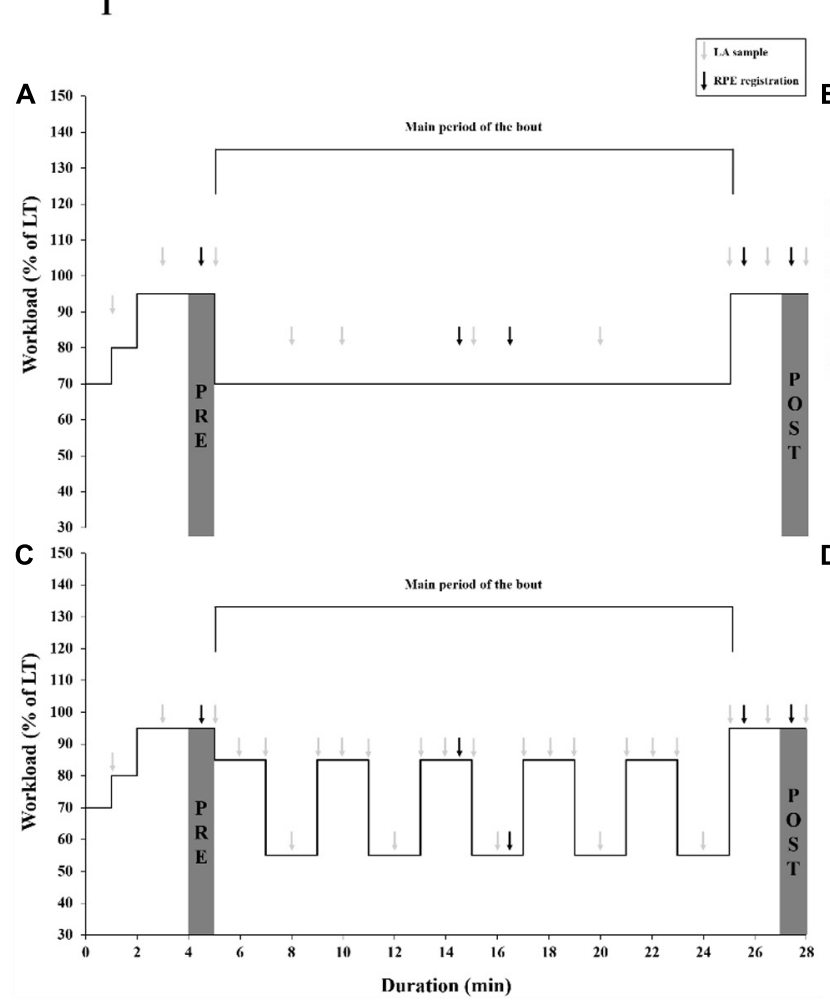

2

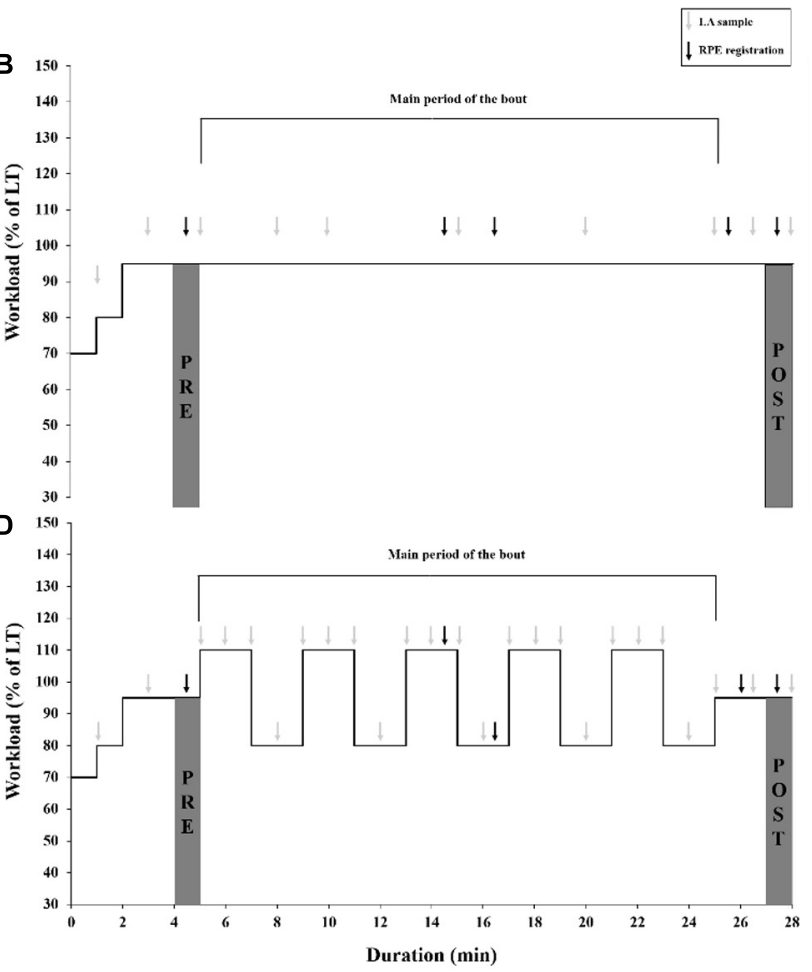

FIGURE 1 | The protocol and design of the four bouts in the present study, (A) low constant (LC), (B) low variable (LV), (C) high constant (HC), (D) high variable (HV), with the incremental ramp of $5 \mathrm{~min}$, the main period of the bout with work-matched VP or CP, and the final 3 min of constant power. Oxygen consumption, respiratory exchange ratio, heart rate, power output and cadence were continuously measured throughout the bout. Gray arrows indicate the time points that LA concentration was measured at. Black arrows indicate the time points that rating of perceived exertion was registered at. The gray bars visualize the time periods used for the PRE (mean during the 5th min for oxygen consumption and heart rate, lactate at the end of the 5th min and rating of perceived exertion registered after $4.5 \mathrm{~min}$ ) and POST (mean during the 28th min for oxygen consumption and heart rate, lactate at the end of the 28 th min and rating of perceived exertion registered after $27.5 \mathrm{~min})$ comparison.

frequently during the VP than CP. The LA data for the CP bouts were interpolated in order to compare the data.

To investigate the effect of the whole 20 -min main period, mean $\mathrm{VO}_{2}, \mathrm{RER}$, and $\mathrm{HR}$ was calculated for the last min prior to the main period, as well as for the last min of the bout (see gray bars representing the PRE and POST periods in Figure 1). RPE for the PRE and POST periods was measured after 4.5 and $27.5 \mathrm{~min}$ and LA was measured at the end of the PRE and POST periods.

Metabolic rate (MR) was calculated from $\mathrm{VO}_{2}$ and RER measurements, converted to energy expenditure (Peronnet and Massicotte, 1991).

\section{Statistical Analysis}

All descriptive data are presented as mean \pm standard deviation. Raw data were visually inspected to check for possible measurement errors prior to further analysis. The main analysis was done using a two-way repeated measures ANOVA to investigate main effects of intensity and power condition as well as to check for possible interaction effects on $\mathrm{VO}_{2}, \mathrm{LA}, \mathrm{HR}$, and RPE. When comparing the PRE to POST values, the delta values were calculated and used in the two-way ANOVA. Where significant effects were found, contrast analysis and pairwise comparisons using Bonferroni correction was used to determine specific effects of intensity and power condition. Strength of the associations in the two-way ANOVA was evaluated using partial eta squared $\left(\eta^{2}\right)$.

Additionally, a paired samples $t$-test was used for comparing values at PRE and POST in terms of $\mathrm{VO}_{2}, \mathrm{LA}, \mathrm{HR}, \mathrm{RER}$, and RPE as well as for investigating segment differences between the two power conditions for both intensities. Statistical significance was accepted at $p<0.05$ and where Bonferroni correction was applied, the corrected alpha is stated. All statistical analyses were conducted using SPSS 25.0 (SPSS, Chicago, United States) for Windows.

\section{RESULTS}

The $\mathrm{VO}_{2}, \mathrm{LA}$, and $\mathrm{HR}$ response during the bouts were significantly affected by power condition and intensity (Figure 2 and Table 2). There was no significant difference in average power output of the $20 \mathrm{~min}$ main period of the bout between $\mathrm{LV}(218 \pm 15 \mathrm{~W})$ and LC $(217 \pm 15 \mathrm{~W})(p=0.43)$, nor between 


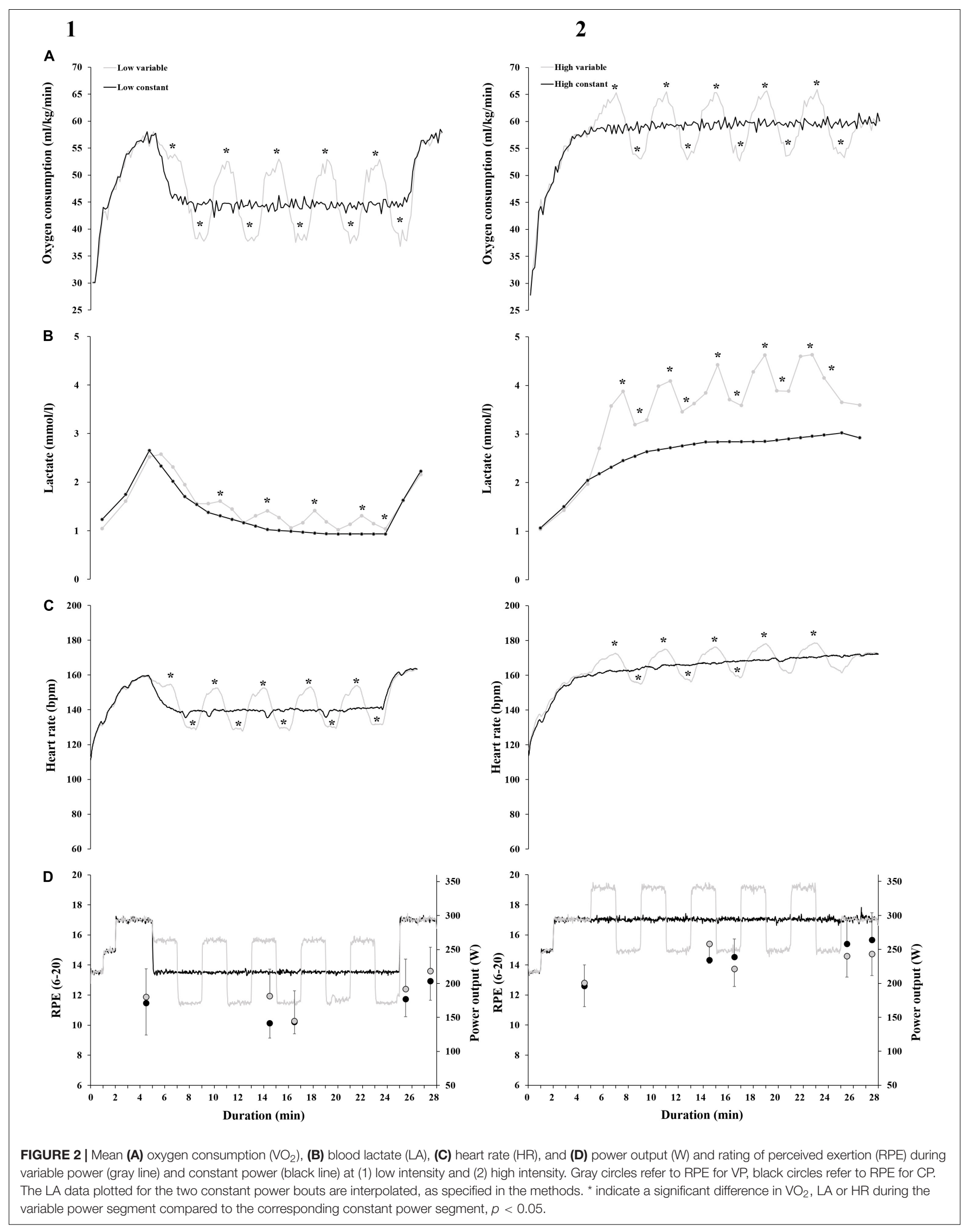


TABLE 2 | Mean \pm standard deviation for different variables during the 20 min main period of the bout for all four bouts.

\begin{tabular}{|c|c|c|c|c|c|c|c|c|c|c|}
\hline & LC & $\mathbf{L V}$ & $\mathrm{HC}$ & HV & Cp & $\mathbf{C} \eta^{2}$ & Ip & $\ln \eta^{2}$ & Intp & $\ln t \eta^{2}$ \\
\hline $\mathrm{VO}_{2}(\mathrm{ml} / \mathrm{kg} / \mathrm{min})$ & $44.9 \pm 3.4$ & $46.0 \pm 3.7^{\star}$ & $59.2 \pm 4.3$ & $59.9 \pm 4.1$ & $<0.05$ & 0.521 & $<0.01$ & 0.990 & 0.33 & 0.068 \\
\hline $\mathrm{LA}(\mathrm{mmol} / \mathrm{l})$ & $1.2 \pm 0.3$ & $1.4 \pm 0.4^{\star}$ & $2.8 \pm 0.8$ & $3.9 \pm 0.7^{\star}$ & $<0.01$ & 0.832 & $<0.01$ & 0.970 & $<0.01$ & 0.668 \\
\hline Heart rate (bpm) & $138 \pm 3.2$ & $139 \pm 9.4$ & $167 \pm 3.0$ & $168 \pm 6.5$ & 0.24 & 0.099 & $<0.01$ & 0.973 & 0.43 & 0.046 \\
\hline RPE $(6-20)^{1}$ & $11.3 \pm 0.7$ & $12.3 \pm 1.6^{\star}$ & $14.9 \pm 1.3$ & $14.9 \pm 1.4$ & 0.09 & 0.192 & $<0.01$ & 0.940 & 0.09 & 0.194 \\
\hline $\operatorname{RER}(-)$ & $0.90 \pm 0.03$ & $0.90 \pm 0.04$ & $0.91 \pm 0.03$ & $0.91 \pm 0.05$ & 0.68 & 0.012 & $<0.01$ & 0.770 & 0.08 & 0.205 \\
\hline $\mathrm{MR}(\mathrm{W})$ & $1153 \pm 81$ & $1174 \pm 79^{\star}$ & $1508 \pm 94$ & $1520 \pm 104$ & $<0.05$ & 0.377 & $<0.01$ & 0.990 & 0.47 & 0.038 \\
\hline
\end{tabular}

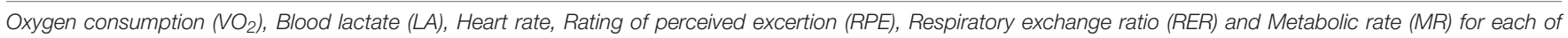

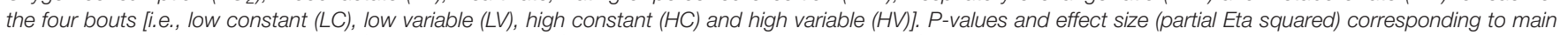

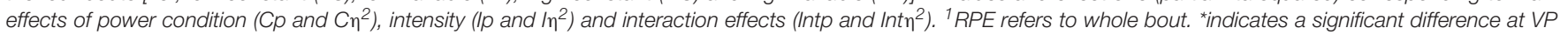
compared to CP at the corresponding intensity evaluated by a t-test, $p<0.05$.

$\mathrm{HV}(294 \pm 20 \mathrm{~W})$ and $\mathrm{HC}(294 \pm 20 \mathrm{~W})(p=0.28)$. Cadence was not different across the four bouts (HC: $87.5 \pm 7.2$, HV: $88.2 \pm 5.0, \mathrm{LC}: 87.2 \pm 5.5, \mathrm{LV}: 87.8 \pm 4.9)$ nor in any PRE to POST comparison (all $\Delta<2.2 \mathrm{rpm}$ and all $p>0.26$ ). Mean $\mathrm{VO}_{2}$ of the bouts corresponded to $63,62,82$, and $81 \%$ of the participants $\mathrm{VO}_{2 \max }$, for $\mathrm{LV}, \mathrm{LC}, \mathrm{HV}$, and $\mathrm{HC}$, respectively.

The differences between the upper and lower power segments of VP and the corresponding segments of CP are visualized in Figure 2. During HV, all segments had a significantly higher LA compared to HC $(p<0.002) .8$ of 10 upper and lower power segments for $\mathrm{HR}$, and 10 of 10 segments for $\mathrm{VO}_{2}$ had significantly higher and lower $\mathrm{VO}_{2}$ and $\mathrm{HR}$, respectively, during $\mathrm{HV}$ compared to the corresponding segments of $\mathrm{HC}$ $(p<0.005)$. Every upper and lower power segment during LV had higher and lower $\mathrm{VO}_{2}$ and $\mathrm{HR}$, respectively, compared to corresponding LC-segments $(p<0.005)$. At low intensity, 4 of 5 upper power segment had significantly higher LA during LV compared to LC $(p<0.002)$. No difference in LA was found when comparing the lower power segments of LV to the same segments of LC, except for the last lower LV-segments, where LA was significantly higher during LV than the corresponding LC-segments $(p<0.002)$. VP had a higher RPE on the upper power segment than the corresponding segment of $\mathrm{CP}$ on both intensities. On the lower power segment, there was no difference in RPE.

The mean $\mathrm{VO}_{2}, \mathrm{LA}, \mathrm{HR}$, whole-bout RPE, RER and MR during the $20 \mathrm{~min}$ main period of the LC, LV, HC, and HV bouts are summarized in Table 2. There was a significant main effect of both intensity and power condition on $\mathrm{VO}_{2}$ and LA (both $p<0.05$, Figure 2). A significant interaction effect between intensity and power condition was found for LA $(p<0.01)$, but not $\mathrm{VO}_{2}(p=0.33)$.

A significant main effect of intensity was found on HR, wholebout RPE and RER (all $p<0.01$, Figure 2). There was a tendency toward a main effect of power condition on whole-bout RPE $(p=0.09)$ as well as a tendency toward an interaction effect between intensity and power condition on whole-bout RPE and $\operatorname{RER}(p=0.09$ and 0.08 , respectively). There was no main effect of power condition for HR or RER (both $p>0.24$ ).

The mean $\mathrm{VO}_{2}$, LA, HR, RPE, RER, and MR during the 3 min of standardized power output prior to (PRE) and after (POST) the LC, LV, HC, and HV bouts with corresponding $p$-values and effect sizes are summarized in Table 3.
There was a main effect of intensity and power strategy on $\mathrm{VO}_{2}, \mathrm{LA}, \mathrm{RER}$, and MR from PRE to POST $(p<0.05) . \mathrm{VO}_{2}$ and $\mathrm{MR}$ increased more following high intensity and CP compared to low intensity and VP, respectively, whereas RER decreased more following high intensity and VP compared to low intensity and $\mathrm{CP}$, respectively. LA decreased following both low intensity bouts. At the high intensity, LA increased following both bouts, and the increase was significantly bigger following VP vs. CP. There was also an interaction effect for LA with a greater effect of power condition at the high intensity compared to low intensity. There was a significant increase in HR from PRE to POST during all of the four bouts $(p<0.05)$, but the change was not significantly different between power conditions. RPE increased significantly from PRE to POST during all of the four bouts $(p<0.05)$, and the increase in RPE following $\mathrm{HC}$ was significantly greater than following $\mathrm{HV}(p<0.05)$.

\section{DISCUSSION}

The aim of the present study was to investigate physiological and perceptual response to $\mathrm{VP}$ vs. $\mathrm{CP}$, and to investigate if variations in power output which span above LT differ from variations below LT. The main finding was that performing the same amount of work with varying power every second minute for $20 \mathrm{~min}$, had a main effect leading to a higher overall oxygen cost, heart rate and blood lactate than maintaining a constant power. At low intensity, the whole-bout RPE corresponded with the physiological parameters and were higher for the VP bout compared to CP. However, whole-bout perceived exertion was similar between the two power conditions at high intensity.

\section{Oxygen Cost}

The finding of a higher oxygen cost during VP compared to $\mathrm{CP}$ at the high intensity, despite matched average work rate, is in contrast to several previous studies on the topic (Liedl et al., 1999; Palmer et al., 1999; Kang et al., 2007; Suriano et al., 2007). However, Suriano et al. (2007) used a lower load and less frequent fluctuations (i.e., $90 \%$ of LT $\pm 20 \%$ every 5 th $\mathrm{min}$ ) and Liedl et al. (1999) used less frequent and lower amplitude fluctuations (1-h maximal power $\pm 5 \%$ every 5 th $\mathrm{min}$ ).

The increases in power output during VP, both below and above the LT will require anaerobic energy contribution due 
TABLE 3 | Change scores \pm standard deviation for different variables from PRE to POST for each of the four bouts.

\begin{tabular}{|c|c|c|c|c|c|c|c|c|c|c|}
\hline & $\Delta \mathrm{LC}$ & $\Delta \mathrm{LV}$ & $\Delta \mathrm{HC}$ & $\Delta \mathrm{HV}$ & $\mathrm{Cp}$ & $\mathbf{C}_{\eta}$ & Ip & In & Intp & Int $\eta$ \\
\hline $\mathrm{VO}_{2}(\mathrm{ml} / \mathrm{kg} / \mathrm{min})$ & $0.5 \pm 1.0$ & $0.3 \pm 1.0$ & $2.7 \pm 1.8^{\star}$ & $1.8 \pm 1.2^{*}$ & $<0.01$ & 0.505 & $<0.05$ & 0.257 & 0.24 & 0.096 \\
\hline LA (mmol/l) & $-0.4 \pm 0.3^{\star}$ & $-0.4 \pm 0.6^{\star}$ & $0.8 \pm 1.0^{\star}$ & $1.7 \pm 0.8^{\star}$ & $<0.05$ & 0.276 & $<0.01$ & 0.826 & $<0.01$ & 0.408 \\
\hline Heart rate (bpm) & $4.4 \pm 2.92^{\star}$ & $3.9 \pm 4.0^{\star}$ & $12.7 \pm 6.3^{\star}$ & $10.6 \pm 7.2^{\star}$ & $<0.01$ & 0.721 & 0.24 & 0.099 & 0.43 & 0.044 \\
\hline RPE $(6-20)^{1}$ & $1.5 \pm 2.5^{\star}$ & $1.7 \pm 1.8^{\star}$ & $3.1 \pm 2.0^{\star}$ & $1.9 \pm 2.1^{\star}$ & 0.06 & 0.237 & 0.16 & 0.139 & 0.06 & 0.221 \\
\hline $\operatorname{RER}(-)$ & $-0.01 \pm 0.01$ & $-0.02 \pm 0.01^{\star}$ & $-0.02 \pm 0.02^{\star}$ & $-0.04 \pm 0.02^{\star}$ & $<0.05$ & 0.539 & $<0.05$ & 0.546 & 0.80 & 0.005 \\
\hline $\mathrm{MR}(\mathrm{W})$ & $13.3 \pm 25.2$ & $-1.15 \pm 21.2$ & $59.6 \pm 48.9^{\star}$ & $38.8 \pm 33.1^{\star}$ & $<0.01$ & 0.443 & $<0.01$ & 0.464 & 0.67 & 0.014 \\
\hline
\end{tabular}

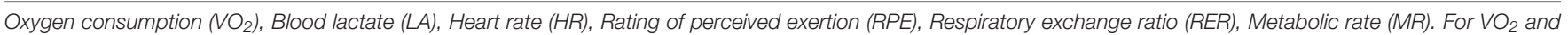

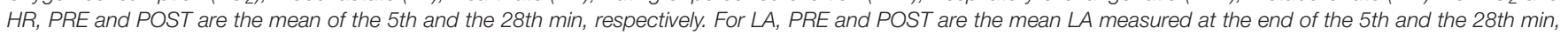

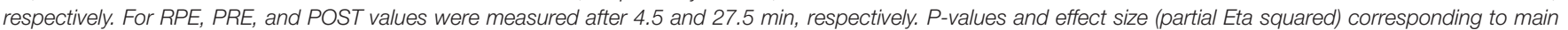

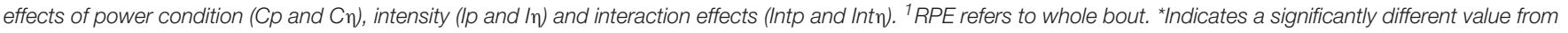
PRE to POST, $p<0.05$.

to the delay in the aerobic system and will also lead to an oxygen debt (Xu and Rhodes, 1999). Acquiring an oxygen debt would lead to an underestimation of the overall oxygen cost as opposed to the increased cost seen in the present study. A greater reduction in exercise intensity will likely allow well trained cyclists to "repay" any potential oxygen debt faster. This can also be substantiated by our findings of higher $\mathrm{VO}_{2}$ during the first upper power segment during the HV bout compared to the corresponding period in $\mathrm{HC}$. This indicates that the participants have fast $\mathrm{VO}_{2}$ kinetics and are able to increase their $\mathrm{VO}_{2}$ rapidly, thus minimizing oxygen debt, a skill useful when racing and attacking. Taken together, this might indicate that an average power output close to LT, with sufficiently large and frequent variations as in the present study may be needed to see an increased oxygen cost.

Exercising above LT also involves a slow component of $\mathrm{VO}_{2}$ (Lucia et al., 2000). The $\mathrm{VO}_{2}$ slow component is reported to be less for professional cyclists than for less trained participants (Lucia et al., 2000, 2002), but given the fluctuation up to $110 \%$ of LT during VP it could possibly be present. Although we found an increase in $\mathrm{VO}_{2}$ through the test, the increase was not different between the power conditions, and thus it does not seem that the slow component can explain the differences between the VP and CP conditions. Additionally, oxygen cost and LA were significantly higher during VP compared to $\mathrm{CP}$ at the low intensity. Any "unpaid" oxygen debt should also be minimal since the exercise intensity was at the most $85 \%$ of LT (Xu and Rhodes, 1999), and thus the magnitude of LA accumulation and the $\mathrm{VO}_{2}$ slow component should be negligible.

\section{Blood Lactate}

As power during $\mathrm{HV}$ fluctuated above and below LT for a prolonged period, the finding of the present study that mean LA was higher for $\mathrm{HV}$ compared to $\mathrm{HC}$, was not surprising, and is in agreement with most of previous studies (Palmer et al., 1999; Suriano et al., 2007; Theurel and Lepers, 2008). LA accumulation increases in an exponential manner when intensity increases (Faude et al., 2009) and thus VP with upper power segments above LT results in periods of rapid increases in blood lactate. The subsequent periods of $80 \%$ of LT results in lactate clearance, but the exponential nature means that the cyclists are able to clear less than they accumulate in the prior segment. Although the average intensity of the high intensity bouts was $95 \%$ of LT, the combination of $10 \mathrm{~min}$ at $110 \%$ of LT and $10 \mathrm{~min}$ at $80 \%$ of LT in the HV bout results in greater blood lactate accumulation compared to HC and over time. Neither Liedl et al. (1999) nor Brickley et al. (2007) found differences in mean LA between VP and CP. However, Liedl et al. (1999) used only 5\% amplitude, and Brickley et al. (2007) used 30 s of $158 \%$ of critical power with 2 min recovery at $73 \%$ of critical power. A power variation amplitude of $5 \%$ may not be enough to elicit an intensity high enough for the exponential aspect of LA accumulation to take full effect, and $30 \mathrm{~s}$ of high power output may not be sufficient duration for LA to accumulate in significant quantity although it may be demanding in other ways.

\section{Metabolic Rate}

The increased LA during VP, taken together with the increased oxygen cost, indicates increased energy requirements. MR was calculated to possibly get a better understanding of the energy requirements of our two power conditions. We found no increase in metabolic rate from PRE to POST during the low intensity bouts. These findings are similar to Hakonssen et al. (2013), who used a maximal and average intensity of $75 \%$ and 55\% of maximal aerobic power, respectively, and is thus comparable to our "low" condition. However, our high intensity conditions produced increases in $\mathrm{MR}$, and a greater increase in MR during the $\mathrm{HC}$ bout compared to the $\mathrm{HV}$ bout, due to a greater decrease in RER values.

The greater decrease in RER values after HV compared to HC indicates a greater reliance on fat metabolism. Cole et al. (2018) demonstrated that carbohydrate feeding can acutely reduce the drop in gross efficiency, and thus increase MR, during an extended cycling bout. Although our participants were able to drink between the two bouts, they could not ingest anything during the 28 min of each bout. Our trial was shorter in duration but higher in intensity, and the increase in fat metabolism indicated by RER values could possibly have produced the same result as demonstrated by Cole et al. (2018). Additionally, when including the finding that the $\mathrm{HV}$ bout produced more lactate than the HC bout, a difference in the anaerobic component between the bouts are present. Although it is difficult to quantify the energy equivalent from blood lactate, it will be greater than 
zero and thus the MR of the HV bout will underestimate the actual energy expenditure.

It should be mentioned that the VP condition ended with a low power output segment prior to the post measurement, which could potentially have led to an underestimation of $\mathrm{VO}_{2}$ and thus MR, due to not fully reaching steady state during the 3-min post period. However, our participants were highly competitive cyclist that are accustomed to power fluctuation and are likely to have fast $\mathrm{VO}_{2}$ kinetics. Additionally, there were no statistically significant difference between the two final $30 \mathrm{~s}$ periods of $\mathrm{VO}_{2}$ and RER measurements or corresponding MR calculations during the 3 -min post period $(p=0.91,0.74$, and 0.84 , respectively).

After considering potential oxygen debt, lactate accumulation and $\mathrm{VO}_{2}$ slow component, we are left with indications that the power fluctuations themselves both require and allows for increased oxygen consumption. Our findings indicate that power fluctuations that reach intensities above steady state differ from fluctuations within steady state.

\section{RPE}

A similar mean RPE-score between VP and CP has been reported previously in the literature (Liedl et al., 1999; Palmer et al., 1999; Bernard et al., 2007; Kang et al., 2007; Lepers et al., 2008), but not when physiological cost differed as in the present study. Generally, it is expected that the whole-bout RPE values coincides with physiological cost, however, the power variations throughout the HV bout introduce frequent changes and this continuous alteration in the task may distract the participants from feeling increased exertion which should correspond to the observed increase in physiological cost. The whole-bout RPE does not seem to be greatly affected by the in-bout RPE which is higher during the upper power segments compared to CP (i.e., at $14.5 \mathrm{~min}$ ) in both the high and low intensity conditions.

The variation resulting from VP could potentially influence RPE by compensating for the seated condition used in the present study. Although cycling position must be standardized because a change from seated to standing can influence physiological cost, a continuous seated position can potentially induce some local fatigue which normally could be alleviated through standing. As different power has been shown to influence both joint power (Skovereng et al., 2015) contribution and muscle activation (Hug and Dorel, 2009), the VP could potentially also alter the muscle contribution and influence the RPE.

Varying power induced higher $\mathrm{VO}_{2}$ and LA at both high and low intensities, but at low intensity, varying power was perceived as more exhausting, with 9 of 15 participants reporting the variable power condition to be more exhausting. To the authors' knowledge, LIT is usually performed with CP among elite competitive cyclists. Consequently, the low intensity bouts with VP in the present study may have been somewhat unfamiliar to the cyclists compared to the high intensity VP bouts, possibly due to VP being more common in a high intensity setting such as interval sessions or racing. Given the large differences in power, comparing the findings at the low intensity in the present study to other studies is difficult. However, as LIT constitutes approximately $80 \%$ of elite cyclists total training volume and also constitutes a large portion of most road cycling mass-starts, these findings could be valuable for athletes and their coaches. As $\mathrm{VO}_{2}$, LA and whole-bout RPE were higher during LV than LC, it appears that the average power output obtained from stochastic low intensity periods of races (or training) cost more, both physiologically and perceptually, than if the same average power output comes from a constant endurance ride in training. However, in this regard, it should be mentioned that we obtained limited RPE measurements during the bouts and focussed on the whole-bout RPE due to practical considerations. In hindsight, additional RPE measurements would have been preferable for comparing with physiological measurements. Indeed, RPE was higher at VP during the upper power segments (i.e., $14.5 \mathrm{~min}$ ). Considering this, a practical implication to coaches and their athletes is that the average numbers, e.g., average power and RPE, don't necessary tell the whole story, and that it is important to add the perceived exertion behind the numbers to the equation.

\section{Upper and Lower Power Segments During VP}

Overall, the segment differences between VP and CP were as expected and in accordance with previous reports (Liedl et al., 1999; Suriano et al., 2007), with higher and lower $\mathrm{VO}_{2}$ and HR values, respectively, during the upper and lower power VPsegments compared to the corresponding CP-segments. Liedl et al. (1999) did not find $\mathrm{VO}_{2}$ to be greater during the upper power VP-segments compared to the corresponding CPsegments, but this may be due to the average of the first and last $75 \mathrm{~s}$ of the segment being used for $\mathrm{VO}_{2}$ calculation and thus potentially underestimating the oxygen cost. Higher LA during the upper power VP-segments compared to the corresponding CP-segments are also reported by Liedl et al. (1999) and Suriano et al. (2007), but these authors reported no difference in LA during the lower power VP-segments compared to the corresponding CP-segments.

\section{Methodological Considerations}

Due to methodological differences in frequency and amplitude of the power variations and mean exercise intensity, the results from the literature are difficult to compare. We decided on our intensity, amplitude and fluctuation frequency based on pilot testing. Our protocol allowed us to reach intensities well above LT (and thus likely above steady state) in the upper power segments of $\mathrm{HV}$ and to achieve a large enough difference between VP and $\mathrm{CP}$ in addition to sufficient time for physiological differences to be detected. Both power variation frequency and amplitude would affect the $\mathrm{LA}$ and $\mathrm{VO}_{2}$ response because of the nature of LA accumulation and $\mathrm{VO}_{2}$ kinetics. Hill and Gibson (2012) compared variations in power output every minute vs. every 5 th min and found no effect on metabolic load. Potentially, the power variation amplitude and the intensity that power is varied around, has a greater effect on the physiological response than the frequency of the variations. Based on pilot testing, we decided that a mean intensity of $95 \%$ of LT was appropriate for the high intensity bout. With this intensity, 15\% fluctuations allowed for an intensity well above LT in the upper power segments of $\mathrm{HV}$ 
(i.e., 110\%). With this protocol, we also achieved a large enough difference between the VP and CP conditions. One could argue that $105 \%$ could be sufficient for exceeding steady state, but based on our pilot testing, we decided that $110 \%$ was feasible without producing too much fatigue.

The performance level of the participants is another methodological consideration that could possibly affect the results. High level cycling races differ from most other endurance sports in terms of frequent and stochastic power variations. The participants in the present study had high $\mathrm{VO}_{2 \max }$ and PPO and were also highly competitive cyclists and thus familiar with intensity fluctuations from their experience racing high level races. Previous studies on power variation have included participants ranging from "healthy, physically active" to "highly trained" cyclists and few include information on racing experience (Palmer et al., 1997), which possibly could impact the outcome of these studies.

\section{Practical Implications}

The VP and CP bouts of this study were designed to replicate a typical interval training session for elite competitive cyclists during their preparation period. The duration of $20 \mathrm{~min}$ that was chosen is also not an uncommon duration for a time trial. Despite the finding of increased oxygen cost and lactate values, RPE was similar between VP and CP at the high intensity. The cyclists were able to complete a total of $10 \mathrm{~min}$ at $110 \%$ of LT during HV with the same RPE-score as riding at a constant power output at $95 \%$ of LT for 20 min during the $\mathrm{HC}$ bout. Considering that VP may, in many cases, be a more race specific training method than CP, utilizing training with VP may be an advantageous and viable option, and we argue that the VP training sessions can be implemented in the daily training of elite cyclists. However, as we demonstrate that there is a significant difference in the physiological cost between VP and CP, coaches should be aware of this difference when designing training programs and calculating the total training load. Furthermore, as the physiological cost of work-matched VP and CP intervals with the same average power seem to differ, it could be hypothesized that when a cyclist performs intervals with spikes only above the average power, the physiological cost will increase even more.

\section{CONCLUSION}

The present study show that varying power for 20 min led to a higher mean oxygen cost, heart rate and lactate than

\section{REFERENCES}

Atkinson, G., and Brunskill, A. (2000). Pacing strategies during a cycling time trial with simulated headwinds and tailwinds. Ergonomics 43, 1449-1460. doi: 10.1080/001401300750003899

Beneke, R. (2003). Methodological aspects of maximal lactate steady stateimplications for performance testing. Eur. J. Appl. Physiol. 89, 95-99. doi: 10.1007/s00421-002-0783-1

Bernard, T., Vercruyssen, F., Mazure, C., Gorce, P., Hausswirth, C., and Brisswalter, J. (2007). Constant versus variable-intensity during cycling: effects maintaining a constant power at the high intensity in a cohort of elite competitive cyclists. These findings were also evident at the low intensity. The perceived whole-bout exertion was higher for VP than $\mathrm{CP}$ at the low intensity, but at the high intensity, it was similar between power conditions, despite a greater in-bout RPE during the high intensity segments. Thus, training with VP seems to be a viable alternative to training with $\mathrm{CP}$, at least at high intensity. Future studies should investigate physiological response to different intensities, amplitudes and possibly a degree of random power variations as seen in cycling races, in addition to longitudinal effects of training with VP vs. CP.

\section{DATA AVAILABILITY STATEMENT}

The raw data supporting the conclusions of this article will be made available by the authors, without undue reservation.

\section{ETHICS STATEMENT}

The studies involving human participants were reviewed and approved by Norwegian Social Science Data Services. The patients/participants provided their written informed consent to participate in this study.

\section{AUTHOR CONTRIBUTIONS}

EK developed the research idea, contributed to the development of study design and protocol, performed the recruitment of participants, the data collection, data inspection, and the data- and statistical analyses, interpreted the results, and wrote the manuscript. KS and GE contributed to the development of study design and protocol, interpretation of the results, data- and statistical analyses, and contributed to revision of the manuscript. All authors contributed to the article and approved the submitted version.

\section{ACKNOWLEDGMENTS}

The authors would like to thank all the participants for their participation and contribution in this study.

on subsequent running performance. Eur. J. Appl. Physiol. 99, 103-111. doi: 10.1007/s00421-006-0321-7

Brickley, G., Green, S., Jenkins, D. G., Mceinery, M., Wishart, C., Doust, J. D., et al. (2007). Muscle metabolism during constant- and alternating-intensity exercise around critical power. Int. J. Sports Med. 28, 300-305. doi: 10.1055/s-2006924354

Cole, M., Hopker, J. G., Wiles, J. D., and Coleman, D. A. (2018). The effects of acute carbohydrate and caffeine feeding strategies on cycling efficiency. J. Sports Sci. 36, 817-823. doi: 10.1080/02640414.2017.13 43956 
Ebert, T. R., Martin, D. T., Stephens, B., and Withers, R. T. (2006). Power output during a professional men's road-cycling tour. Int. J. Sports Physiol. Perform. 1, 324-335. doi: 10.1123/ijspp.1.4.324

Faria, E. W., Parker, D. L., and Faria, I. E. (2005). The science of cycling: physiology and training - part 1. Sports Med. 35, 285-312. doi: 10.2165/00007256200535040-00002

Faude, O., Kindermann, W., and Meyer, T. (2009). Lactate threshold concepts: how valid are they? Sports Med. 39, 469-490. doi: 10.2165/00007256-20093906000003

Foster, C., Snyder, A. C., Thompson, N. N., Green, M. A., Foley, M., and Schrager, M. (1993). Effect of pacing strategy on cycle time trial performance. Med. Sci. Sports Exerc. 25, 383-388.

Haakonssen, E. C., Martin, D. T., Burke, L. M., and Jenkins, D. G. (2013). Energy expenditure of constant- and variable-intensity cycling: power meter estimates. Med. Sci. Sports Exerc. 45, 1833-1840. doi: 10.1249/mss.0b013e31828e18e6

Hill, C. F., and Gibson, A. (2012). The effect of power alternation frequency during cycling on metabolic load and subsequent running performance. J. Sci. Cycling $1,35-41$.

Hug, F., and Dorel, S. (2009). Electromyographic analysis of pedaling: a review. J. Electromyogr. Kinesiol. 19, 182-198. doi: 10.1016/j.jelekin.2007.10.010

Kang, J., Mangine, G. T., Ratamess, N. A., Faigenbaum, A. D., and Hoffman, J. R. (2007). Influence of intensity fluctuation on exercise metabolism. Eur. J. Appl. Physiol. 100, 253-260. doi: 10.1007/s00421-007-0424-9

Lepers, R., Theurel, J., Hausswirth, C., and Bernard, T. (2008). Neuromuscular fatigue following constant versus variable-intensity endurance cycling in triathletes. J. Sci. Med. Sport 11, 381-389. doi: 10.1016/j.jsams.2007.03.001

Liedl, M. A., Swain, D. P., and Branch, J. D. (1999). Physiological effects of constant versus variable power during endurance cycling. Med. Sci. Sports Exerc. 31, 1472-1477.

Lucia, A., Hoyos, J., and Chicharro, J. L. (2000). The slow component of VO2 in professional cyclists. Br. J. Sports Med. 34, 367-374. doi: 10.1136/bjsm.34.5.367

Lucia, A., Hoyos, J., Santalla, A., Pèrez, M., and Chicharro, J. L. (2002). Kinetics of $\mathrm{VO}(2)$ in professional cyclists. Med. Sci. Sports Exerc. 34, 320-325.

Palmer, G. S., Borghouts, L. B., Noakes, T. D., and Hawley, J. A. (1999). Metabolic and performance responses to constant-load vs. variable-intensity exercise in trained cyclists. J. Appl. Physiol. 87, 1186-1196. doi: 10.1152/jappl.1999.87.3. 1186

Palmer, G. S., Hawley, J. A., Dennis, S. C., and Noakes, T. D. (1994). Heart rate responses during a 4-d cycle stage race. Med. Sci. Sports Exerc. 26, 1278-1283.

Palmer, G. S., Noakes, T. D., and Hawley, J. A. (1997). Effects of steady-state versus stochastic exercise on subsequent cycling performance. Med. Sci. Sports Exerc. 29, 684-687. doi: 10.1097/00005768-199705000-00015

Peronnet, F., and Massicotte, D. (1991). Table of nonprotein respiratory quotient an update. Can. J. Sport Sci. Rev. Can. Des Sci. Sport 16, 23-29.
Rønnestad, B. R., and Hansen, J. (2018). A scientific approach to improve physiological capacity of an elite cyclist. Int. J. Sports Physiol. Perform. 13, 390-393. doi: 10.1123/ijspp.2017-0228

Seiler, S. (2010). What is best practice for training intensity and duration distribution in endurance athletes? Int. J. Sports Physiol. Perform. 5, 276-291. doi: $10.1123 /$ ijspp.5.3.276

Seiler, S., and Tønnesen, E. (2009). Intervals, threshold, and long slow distance: the role of intensity and duration in endurance training. Sport Sci. 13, 32-53.

Skovereng, K., Ettema, G., and Van Beekvelt, M. (2015). Local muscle oxygen consumption related to external and joint specific power. Hum. Mov. Sci. 45, 161-171. doi: 10.1016/j.humov.2015.11.009

Solli, G. S., Tønnesen, E., and Sandbakk, $\varnothing$ (2017). The training characteristics of the world's most succesful female cross-country skier. Front. Physiol. 8:1069. doi: 10.3389/fphys.2017.01069

Suriano, R., Vercruyssen, F., Bishop, D., and Brisswalter, J. (2007). Variable power output during cycling improves subsequent treadmill run time to exhaustion. J. Sci. Med. Sport 10, 244-251. doi: 10.1016/j.jsams.2006. 06.019

Swain, D. P. (1997). A model for optimizing cycling performance by varying power on hills and in wind. Med. Sci. Sports Exerc. 29, 1104-1108. doi: 10.1097/ 00005768-199708000-00017

Sylta, O., Tonnessen, E., Hammarstrom, D., Danielsen, J., Skovereng, K., Ravn, T., et al. (2016). The effect of different high-intensity periodization models on endurance adaptations. Med. Sci. Sports Exerc. 48, 2165-2174. doi: 10.1249/ mss.0000000000001007

Theurel, J., and Lepers, R. (2008). Neuromuscular fatigue is greater following highly variable versus constant intensity endurance cycling. Eur. J. Appl. Physiol. 103, 461-468. doi: 10.1007/s00421-008-0738-2

Wells, M. S., and Marwood, S. (2016). Effects of power variation on cycle performance during simulated hilly time-trials. Eur. J. Sport Sci. 16, 912-918. doi: 10.1080/17461391.2016.1156162

$\mathrm{Xu}, \mathrm{F}$., and Rhodes, E. C. (1999). Oxygen uptake kinetics during exercise. Sports Med. 27, 313-327. doi: 10.2165/00007256-199927050-00003

Conflict of Interest: The authors declare that the research was conducted in the absence of any commercial or financial relationships that could be construed as a potential conflict of interest.

Copyright (c) 2020 Kolsung, Ettema and Skovereng. This is an open-access article distributed under the terms of the Creative Commons Attribution License (CC BY). The use, distribution or reproduction in other forums is permitted, provided the original author(s) and the copyright owner(s) are credited and that the original publication in this journal is cited, in accordance with accepted academic practice. No use, distribution or reproduction is permitted which does not comply with these terms. 